\title{
Nuevos rumbos del comparatismo: literatura comparada y literatura-mundo
}

\author{
Eduardo Coutinho ${ }^{\bullet}$
}

Universidade Federal do Rio de Janeiro

\begin{abstract}
Resumen
Desde su configuración y consolidación como disciplina académica, en la segunda mitad del siglo XIX, la Literatura Comparada ha pasado por significativas transformaciones, $y$ hoy día, lejos de atenerse a los estudios de carácter binario entre autores, obras o movimientos literarios o al canon de la tradición occidental, la disciplina se encuentra abierta a todo tipo de expresión literaria y cultural y receptiva a otras áreas del conocimiento, caracterizándose como un verdadero diálogo de culturas. Entre los cambios por los que ha pasado el comparatismo, destacamos en este ensayo la importancia que pasó a ser dada a la circulación de la obra literaria, marcada por una mayor receptividad respecto al estudio de obras en traducción y por el énfasis sobre su contexto de recepción, y que ha dado origen a toda una línea de investigación del comparatismo que se designó «literatura-mundo». En este texto haremos una reflexión sobre esas transformaciones por las que ha pasado el comparatismo y procuraremos mostrar que, lejos de estar debilitada, la disciplina se encuentra en plena revitalización.
\end{abstract}

\section{Palabras clave}

- Literatura comparada · literatura mundo · nuevos rumbos

\footnotetext{
- Eduardo F. Coutinho (PhD-U.C.Berkeley) es Profesor Titular Emérito de Literatura Comparada de la Universidade Federal do Rio de Janeiro. Fue Profesor Visitante en universidades en varios países y Distinguished Visiting Scholar en la Universidad de Illinois, EUA. Fue miembro fundador y Presidente de la Asociación Brasileña de Literatura Comparada y Vice-Presidente de la Asociación Internacional de Literatura Comparada. Entre sus libros destácanse: The Synthesis Novel in Latin America, Em busca da terceira margem, Literatura Comparada na América Latina (trad. al esp.), Literatura Comparada: reflexões (trad. al esp.), y Rompendo barreiras: ensaios.
} 


\section{Abstract}

Since its inception and consolidation as an academic discipline in the second half of the nineteenth century, the field of comparative literature has undergone significant transformations. Far from ascribing to binary studies around the work of authors, literary masterpieces or approaches or the Western canon, this discipline has remained open to all kinds of literary and cultural manifestations and receptive of other knowledge areas, thus becoming a true dialogue amongst cultures. Among the changes that the scope of comparative literature has experienced, we hereby highlight the "circulation" of a given literary masterpiece, often characterized by a good reception on the part of translation studies and by the emphasis placed on its reception context; which in tandem has given birth to a research arena within this field that is known as "world literature». In this text, we will attempt to reflect upon the aforementioned transformations regarding the spectrum of comparative literature and try to demonstrate that far from being forgotten, the discipline has been completely revitalized.

\section{Key words}

· Comparative literature $\cdot$ world literature $\cdot$ new paths

En un libro publicado en 2003 con el título de Death of a Discipline, Gayatri Spivak hace un inventario de la Literatura Comparada como disciplina académica afirmando que ella había dejado de existir en su sentido tradicional, y reclama la necesidad de un nuevo comparatismo que tenga en consideración las diferencias idiomáticas, el poder estético de la literatura y las consecuencias políticas de leerse y escribirse sobre obras de otras culturas (Spivak, 2003). Como podemos ver, el término death, presente en el título, porta no sólo la significación de «muerte» $\mathrm{O}$ "final», sino principalmente la de «recomienzo", de "comienzo de un nuevo ciclo», lo que nos transporta a las transformaciones por las que la disciplina ha pasado desde su configuración y consolidación. Ya en el año de 1958, durante el Congreso de la Asociación Internacional de Literatura Comparada, René Wellek había hecho referencia a la crisis de la disciplina en un texto que ha causado fuerte polémica y que ha dado origen a la designación de «escuelas» para sus diferentes momentos (Coutinho \& Carvalhal, 2011). Pocos ańos después, en conformidad con los estatutos de la Asociación Norteamericana, concebida bajo el modelo de 
la Asociación Internacional, surge el primer informe sobre el estado de la disciplina, al cual siguieron otros cuatro, con un intervalo de una década entre cada uno de ellos. Estos informes, el último de los cuales publicado en 2017, aunque vueltos hacia el medio académico norteamericano, reflejan los cambios ocurridos en el seno de la disciplina y la manera como sus especialistas la veían, ofreciendo en consecuencia un cuadro de su evolución.

Los dos primeros informes, que traen las fechas de 1965 y 1975, constituyeron una especie de sumario de las normas que la Literatura Comparada debería respetar y de los límites que ella debería observar, sobre todo respecto a la habilidad lingüística del especialista y a las relaciones de la disciplina con las demás manifestaciones artísticas y con otras áreas del conocimiento (Berheimer, 1995). Se defendía en esos informes la necesidad de utilización de textos en su idioma original, incluso en las clases, el dominio de las llamadas «grandes obras» de la tradición occidental y de la historia de esa tradición, y la familiaridad con las principales corrientes teórico-críticas del pensamiento y con los diversos métodos de abordaje del fenómeno literario. Han sido muy criticados los cursos basados en obras traducidas, especialmente los de postgrado, y la interdisciplinariedad, aunque estimulada de modo general, ha sido tratada con desconfianza. Se criticó también el énfasis sobre la teoría que pasó a dominar los estudios de Literatura Comparada desde la década de 1970, confiriendo al estudio de la disciplina un carácter predominantemente sincrónico. Este dominio de la teoría, que alcanzó un nivel sin precedentes en un país que siempre se ha caracterizado por una visión de mundo más pragmática, derivó del respaldo que la teoría francesa post-estructuralista vino a dar a los movimientos de orden político de los llamados "grupos minoritarios», y constituye la base del informe siguiente, surgido en 1993, después de un hiato en la década de 1980.

Este informe, coordinado por Charles Berheimer, tuvo como eje la cuestión de cómo la Literatura Comparada debería posicionarse delante del espacio que la teoría estaba ocupando en el contexto norteamericano, como también delante de los Estudios Culturales en boga en el momento y de la mayor amplitud de los estudios literarios en sus dimensiones multicultural, global e interdisciplinaria. El aparente internacionalismo defendido por el comparatismo del periodo post-guerra fue revelado como eurocéntrico y la tentativa de establecimiento de un modelo para la enseńanza de la disciplina había perdido su eficacia frente a la porosidad cada vez mayor de la práctica académica. El comparatismo pasó a incluir productos estéticos normalmente estudiados por otras disciplinas o provenientes de contextos culturales diversos - de carácter erudito o popular, colonial o postcolonial- y estudios de género y de modos de representación de grupos étnicos distintos, como también discusiones sobre la función y el valor de los propios estudios literarios. El informe se distingue de los anteriores por incluir textos de diversos investigadores sobre su propia visión de la disciplina y, en vez de constituir un conjunto de diagnósticos y de tentativas de regularización, se erige como un diálogo vivo y a veces polémico sobre el asunto. Este modelo es el que se encuentra también en la base del informe siguiente, de Haun Saussy, que examinó a la disciplina a la luz de los cambios geopolíticos ocurridos en el principio del siglo XXI (Saussy, 2006).

Tanto el informe de Bernheimer como el de Saussy indican una significativa ampliación de la esfera de los estudios de Literatura Comparada. Pero mientras el 
primero se restringe al carácter inclusivo de la disciplina, que abarca un número cada vez mayor de literaturas nacionales, de macro o micro-regiones distintas o provenientes de grupos minoritarios o de expresiones culturales dentro de un solo contexto, el segundo seńala un cambio de paradigma, sobre todo respecto al énfasis excesivo que estaba siendo puesto sobre la teoría en el medio académico, a punto de causar un cierto alejamiento del estudio del texto literario. En palabras del propio Saussy en un ensayo incluido en ese volumen, «en algunos momentos, en la última década, se ha vuelto posible realizar una carrera en los estudios literarios sin hacerse referencias concretas a obras de literatura» (Saussy, 2006: 12). Esa fiebre de la teoría que ha dominado el medio académico norteamericano en las últimas décadas del siglo XX trajo sin duda contribuciones relevantes para los estudios literarios, sobre todo por la diseminación del pensamiento de los filósofos post-estructuralistas franceses que resultó en lo que vino a ser designado Desconstrucción en Estados Unidos. Pero su exceso ha dado origen también a reacciones, entre las cuales la propuesta de la World Literature, que ha llamado atención hacia la necesidad de retorno a los textos literarios y en una escala mucho más amplia que anteriormente.

Ese alejamiento del texto literario, causado por el énfasis excesivo en la Teoría, tuvo también un correlato en la ola que los Estudios Culturales ha desencadenado en el contexto norteamericano de la década de 1990, y las reacciones en defensa del texto ya se hallan mencionadas en los informes de Bernheimer y de Saussy. Con todo, en ese sentido, tanto los organizadores de los volúmenes como algunos de los colaboradores, además de señalar la importancia de las contribuciones de esa corriente de pensamiento para los Estudios Literarios, sobre todo por su carácter inclusivo que pasó a contemplar textos culturales distintos, dejan claro que ella no ha negado jamás, como algunos de sus seguidores llegaron a creer, la especificidad de la obra literaria. El agrandamiento del ámbito de los Estudios Literarios ha proporcionado a los comparatistas una articulación renovada del valor de la obra de arte respecto tanto a sus aspectos individuales, subjetivos, como a sus implicaciones de orden social o político, y vino a demostrar que esos dos rasgos, lejos de ser antagónicos, se encuentran inevitablemente asociados. La Literatura Comparada está vinculada tanto con la especificidad como con el elemento relacional: de un lado ella busca escudriñar la especificidad de su objeto, la obra literaria, y de otro sus relaciones con otras formas de expresión y con los discursos que constantemente se crean sobre ella.

El informe presentado en 2017 y coordinado por Úrsula Heise (Heise, 2017), se compone también de textos de diferentes especialistas, sumados a algunas entrevistas y a breves tentativas de resemantización de ciertos conceptos básicos de la disciplina, y deja claro que actualmente tanto los estudios literarios de modo general como la Literatura Comparada en particular sólo pueden ser comprendidos como un conjunto de abordajes teóricos y analíticos sobre cuestiones de lenguaje, de literatura y de medios. Es solamente desde el punto de vista de esa constelación de abordajes que se puede trazar una cartografía del comparatismo: sus principales implicaciones teóricas y metodológicas, sus negociaciones con las escalas globales, regionales, nacionales y locales de producción y recepción de textos, su relación con los medios antiguos y recientes, y su posición en el conjunto de nuevas áreas de investigación interdisciplinarias, muchas de las cuales relacionadas con la ciencia y la tecnología. Entre las diversas cuestiones tratadas en el informe que están 
ocupando un espacio cada vez mayor en la agenda de la Literatura Comparada en las últimas décadas se encuentra la que ha sido designada de World Literature, es decir, de "literatura-mundo»" a veces también referida como «literatura mundial» o «literatura global», y que fue designada por Franco Moretti como «lectura distante» ${ }^{2}$ (Moretti, 2013) y definida posteriormente por David Damrosch como "un modo de circulación y de lectura» (Damrosch, 2003), una manera de leer que confiere atención especial a la recepción de un texto más allá de su cultura de origen.

El término Weltliteratur fue creado por Goethe en la primera mitad del siglo XIX y difundido por Eckermann, después de la publicación de su Gespräche mit Goethe in den letzten Jahren seines Lebens, en 1835 — traducida al inglés como Conversations with Eckermann, donde el organizador del volumen ha puesto a Goethe, en vez de Eckermann, como autor. Reaccionando contra la idea de literatura nacional de moda en aquella época, Goethe afirmaba que la literatura era una producción del hombre que pertenecía a toda la humanidad, y que por tanto no podría restringirse a fronteras (Damrosch, 2003). Años después, en 1847, Marx y Engels adoptaron el término de Goethe al afirmar que desde las muchas literaturas nacionales o locales ha surgido la «literatura del mundo» o «literatura mundial», un patrimonio de toda la humanidad (Damrosch, 2003). Sin embargo, desde el principio el término ha presentado problemas por causa de la imposibilidad de definirse con exactitud. ¿Qué sería una «literatura mundial»? ¿La suma de toda la producción literaria del mundo en todos los tiempos y lugares? ¿Un canon? ¿Pero cómo un canon, si la idea de canon implica un recorte? ¿Qué recorte entonces se debe adoptar? ¿Cómo privilegiar determinadas obras en detrimento de otras y en qué criterios nos basaríamos para establecer eso? ¿Lo estético? ¿Pero qué sería lo estético? Y así como en el caso de la producción, o del corpus, en el caso de la reflexión sobre la literatura el término también se ha problematizado y adquirió muchas veces sentidos bastante distintos. En la esfera de la Literatura Comparada como disciplina académica, ha sido a veces empleado como sinónimo de «literatura general» o como una de las dimensiones del comparatismo, que se oponía a la tradición occidental y que se ocupaba de las relaciones entre las producciones literarias de Occidente y de Oriente, y, a mediados del siglo XX, el carácter cosmopolita presente en Goethe ha sido confundido con la búsqueda de universales, dominante entonces, pasando a asumir un tono hegemónico no sólo distante como también muy distinto del sentido original del término.

En los últimos años, sin embargo, el término ha pasado por un proceso de resemantización y, aunque siga siendo empleado para referirse a obras de todo el mundo, no se aplica más a un canon inaccesible de textos. Al contrario, su sentido se restringe ahora a las obras literarias que circulan más allá de su cultura de origen, ya sea en traducción como en su idioma original. El concepto pasa a significar un modo de circulación y de lectura de textos, aplicable tanto a obras individuales como a conjuntos determinados de obras que oscilan de acuerdo con circunstancias históricas y culturales distintas. Una obra puede pertenecer o dejar de pertenecer al canon de la literatura mundial por un doble proceso, como afirma David Damrosch: primero por ser leída como literatura, y, además, por circular en una instancia más amplia que su local lingüística y cultural (Damrosch, 2003). Una obra puede también, a lo largo de los tiempos, entrar y salir muchas veces de este canon, y puede funcionar, en un cierto momento, como literatura-mundo para algunos lectores y no para otros, así como para algunos tipos de lectura y no para 
otros. Ese cambio por el cual la obra puede pasar deriva no de un proceso interno, sino de una dinámica de transformación o de contestación cultural. Pocas obras mantienen un lugar en el conjunto de obras-maestras de la tradición mundial.

La noción de circulación de una obra más allá de su contexto de origen constituye un elemento-clave en su configuración como "literatura mundo», pero en la reflexión de algunos especialistas eso no basta para caracterizarla como tal. Es necesario, para ellos, que la obra — como también el conjunto de obras o el género- sea leída como una expresión literaria, es decir, que ella sea tomada en sus componentes estéticos, y la medida de esos componentes es conferida por la crítica. Es la crítica literaria la que delimitará los elementos estéticos de cada texto y configurará el canon de una cultura, y es ella también la que esbozará el canon de la literatura mundial con base en los cánones de las diversas culturas. Este último se instituye como una selección hecha desde los cánones de las diversas culturas que forman esa entidad abstracta designada mundo, y tiene como rasgo fundamental su carácter inclusivo. Este rasgo constituye un gran avance en los estudios de Literatura Comparada porque rompe con el etnocentrismo que limitaba el canon global a obras europeas, y más recientemente también norteamericanas, pero presenta por otro lado como problema una ausencia de cuestionamiento de la propia noción de canon y de los procesos utilizados en su constitución. Esta es una de las divergencias, por ejemplo, entre los defensores de la propuesta y los críticos post-modernos o post-coloniales, que optan por una posible descanonización.

Este nuevo concepto de «literatura-mundo» se origina en gran parte de la importancia que pasó a ser conferida en las últimas décadas a dos cuestiones íntimamente relacionadas: la traducción de obras literarias y el contexto de recepción de esas obras. Desde el principio de los estudios de Literatura Comparada siempre hubo una fuerte reacción a la lectura de obras en traducción, y como los comparatistas eran generalmente versados en más de un idioma, y a veces en varios, eso nunca ha constituido un impedimento para el desarrollo de la disciplina. Al contrario, era un aspecto favorable que le confería prestigio y un puesto seguro en la esfera de las Humanidades en general. Hasta las décadas de 1970 y 1980, la lectura de obras literarias en traducción solo estaba permitida en los cursos de graduación, como atestiguan los primeros informes de la Asociación Norteamericana, o en los casos en que el estudiante estaba especializándose en una literatura considerada exótica, lo que constituía un rasgo evidente de etnocentrismo. Sin embargo, esa exigencia pasó a ser cuestionada por su carácter elitista y, con los avances de los estudios de Traducción, que han tenido como corolario una ampliación del concepto en las últimas décadas, la cuestión se ha consolidado, y la lectura en traducción pasó a conquistar un importante espacio en el medio académico. En el caso de la «literatura-mundo», la cuestión fue todavía más lejos: como era necesario que la obra literaria, para poder circular más allá de su contexto de origen, tuviera que ser traducida, ya que la mayoría de la gente no lee literatura extranjera en lengua original, la traducción pasó a ser parte directamente del concepto. De ese modo, en vez de ser considerada como una producción menor, la obra traducida pasó a ser vista ella propia como un elemento del sistema de la literatura-mundo. Eso no quiere decir, con todo, que el interés por el estudio de las lenguas haya sido abandonado o relegado a un plano secundario.

Hoy día es de conocimiento general que el contexto de recepción de una obra literaria pasó a ocupar un primer plano en los estudios de Literatura Comparada 
desde la Escuela de Constanza de Estética de la Recepción. Pero no se puede dejar de señalar que una década antes del desarrollo de esta corriente teórico-crítica Robert Escarpit ya había realizado en Francia estudios importantes sobre el rol del público lector. Desde entonces, la figura del lector nunca más ha perdido su relevancia, y el contexto de recepción se ha vuelto tan importante como la propia obra y su contexto de producción. En el caso de la literatura-mundo, en especial, esa figura constituye un elemento clave ya que se trata fundamentalmente de un estudio de circulación y de lectura de obras. La obra literaria adquiere vida nueva en el momento en que ingresa en el seno de la literatura-mundo, es decir, cuando ella traspasa sus fronteras nacionales y/o idiomáticas, y penetra en la esfera de otros sistemas literarios y estéticos; en otras palabras, ella se vuelve una obra de literatura-mundo cuando pasa a circular más allá de su cultura originaria, sea en traducción sea en su idioma primero. La Eneida, de Virgilio, por ejemplo, fue largamente leída en puntos diferentes de Europa en el propio idioma en el que fue escrita —el latín-, y varios textos de Edgard Allan Poe pasaron a ser vistos como obras-maestras desde el momento en el que han sido traducidos al francés por Baudelaire.

La literatura-mundo puede configurarse, según algunos comparatistas, entre los cuales David Damrosch, desde perspectivas distintas: primero como un conjunto de obras clásicas; en seguida como un canon evolutivo compuesto de obras-maestras, y por último como "ventanas hacia el mundo» (Damrosch, 2003: 15). Las obras clásicas son las de valor trascendental o fundacional, como las que, en la tradición occidental, son identificadas con la producción griega o romana. Las obras-maestras, a diferencia de las primeras, pueden ser antiguas o modernas y no tienen necesariamente un valor fundacional. Y las «ventanas hacia el mundo» son obras que, independientemente de su carácter estético, actúan sobre sus contextos de recepción y ofrecen importantes contribuciones para la visión de mundo dominante en esos contextos y para sus relaciones interculturales con otros contextos. Esas tres perspectivas no son excluyentes: una obra como la Eneida puede ser al mismo tiempo un clásico, una obra-maestra y una ventana hacia el mundo contemporáneo de Virgilio, pero en todos esos casos ellas juegan un rol fundamental en la ampliación o la reformulación de los cánones tradicionalmente presentes en el medio académico. Los cursos de literatura-mundo son en general multitemporales y multiculturales, y pueden abarcar obras provenientes de tiempos y lugares bastante distintos, incluso producidos en una expresión lingüística dejada a la orilla, como el libro Me llamo Rigoberta Menchú.

Esta visión de la literatura-mundo como un paisaje amplio y abarcador ha sido apreciado tanto por los comparatistas que están en búsqueda de una escala planetaria como reacción a todo tipo de etnocentrismo como por los que han criticado el globalismo por sus implicaciones de orden imperialista o por relegar a un segundo plano los estudios de literatura más centrados en el texto. Sin embargo, este nuevo paradigma ha portado también algunos problemas, como el de la mediación entre un abordaje de carácter más genérico de la literatura y otro con un sentido más atomístico como el que los norteamericanos han designado de close reading, presente, por ejemplo, en las corrientes llamadas inmanentistas o intrínsecas. Una solución posible mencionada por algunos investigadores consiste en el abandono de cualquier perspectiva dicotómica como la que opone una sistematización globalizadora y la diversidad y especificidad de los textos escogidos. Eso se vuelve todavía más relevante cuando nos acordamos que los cánones de la literatura mundial, 
como los de los estudios literarios en general, están basados en intereses de grupos determinados, que ejercen también influencia sobre la manera como ellos son traducidos, comercializados y leídos. En India, por ejemplo, la literatura-mundo adquiere una forma específica como consecuencia de la multiplicidad lingüística del país y de la presencia fuerte del inglés desde el proceso de colonización.

Sin embargo, en todos esos casos, la perspectiva adoptada por el comparatista está siempre condicionada a su locus de enunciación, porque los patrones globales de circulación de la literatura-mundo toman forma en sus manifestaciones locales. En América Latina, por ejemplo, no fueron pocas las obras que, por la circulación que han tenido en otros contextos, integraron muchas veces un sistema de literatura-mundo construido fuera del continente. Es el caso de la obra de Borges o de García Márquez, que constituyeron objeto de estudio en muchos centros académicos tanto europeos como norteamericanos. Recuérdese que la primera ha sido incluso mencionada por Foucault como una de las principales motivaciones para la creación de su libro Les mots et les choses, y que la segunda fue en gran parte responsable por la diseminación del concepto de «realismo maravilloso» tomado de Carpentier. Es el caso también de novelas como D. Casmurro, de Machado de Assis, cuya reflexión de la scholar norteamericana Helen Caldwell vino a modificar la lectura crítica de la obra realizada en Brasil. Menciónense todavía obras como la de Clarice Lispector, que pasó a integrar un número expresivo de cursos sobre la producción femenina enseñados en universidades europeas y norteamericanas, o la de Guimarães Rosa, que pasó a ser vista como fundamental en los estudios de literatura de expresión portuguesa en el continente africano.

El más reciente informe, con fecha de 2017 y coordenado por Úrsula Heise, da continuidad al modelo de los dos anteriores, pero añade a los textos seleccionados breves reflexiones sobre algunos términos fundamentales de la Literatura Comparada, ensayos sobre la práctica y el futuro de la disciplina, comentarios sobre sus nuevos y antiguos paradigmas y entrevistas a docentes e investigadores del área (Heise, 2017). Esos elementos adicionales, con excepción de las entrevistas, han sido seleccionados de un website, creado por la Asociación con el objetivo de reunir opiniones y posicionamientos variados sobre el estado actual del comparatismo. Y lo que se destaca de todo ese esfuerzo es, como hemos afirmado, el amplio abanico de posibilidades que la Literatura Comparada abarca actualmente: sus relaciones con las principales corrientes teórico-críticas, sus maneras de abordar las dimensiones locales, regionales, nacionales y globales de la producción y recepción de textos, su interrelación con las nuevas formas de actuación mediática y su relación recíproca con las nuevas áreas interdisciplinarias de investigación, que incluyen la ciencia y la tecnología. Explotar las preocupaciones del comparatismo en los estudios de nuevas culturas de los medios, como también en áreas más recientes de investigación, entre las cuales la Ecología Social, las Ciencias Ambientales y los Derechos Humanos, ha sido uno de los principales desafíos de la disciplina, y en todos esos casos se destaca la contribución al campo de las Humanidades en general.

La permeabilidad de la Literatura Comparada, derivada de su carácter de transversalidad, que la ha vuelto una disciplina siempre propensa al diálogo, fue tal vez el aspecto que más ha contribuido para su reformulación y ampliación. Y esta reformulación ha sido tan expresiva desde la segunda mitad del siglo $\mathrm{XX}$ al presente que se vuelve imposible afirmar que el área de estudios estaría en vías de desaparición. El título del libro de Gayatri Spivak que mencionamos al principio 
de este texto no constituye una señal de luto, sino una promesa de recomienzo, como ha observado Jean Franco, en un comentario crítico (en Spivak, 2003). Se trata de una obra visionaria, que señala una transformación de la disciplina, una extraordinaria apertura, difícil de observarse en otras esferas del conocimiento. La Literatura Comparada cuyo óbito podemos celebrar es la que se limitaba a la perspectiva binaria dominante hasta mediados del siglo XX, en sus formas puramente historicista o formalista. Desde aquel momento hacia el presente, sin embargo, la disciplina ha evolucionado de tal modo que, lejos de anunciarse su extinción, lo que se puede registrar con seguridad es su extraordinario reflorecimiento.

\section{Notas}

${ }^{1}$ En su ensayo «Inventar a ler. Literatura-mundo em portugués», Helena Buescu hace una distinción entre «literatura-mundo» $\mathrm{y}$ «literatura mundial», y opta por la utilización del primer término por lo siguiente: «La opción por literatura-mundo significa una forma distinta de concebir la expresión mundial o planetaria de la literatura, una expresión que es al mismo tiempo dilatada (o sea, potencialmente planetaria) sin que con eso se busque cualquier efecto de agotamiento, conclusión, encierro o simple representación cuantitativa» (en Abdala Junior, 2014: 45-46). Por eso, optamos también por utilizar el mismo término en este ensayo. Hay que señalar que el término está siendo empleado también con sentido semejante en el universo de expresión francesa.

${ }^{2}$ El concepto morettiano de «distant Reading» refiere más específicamente a un modo de abordar a la producción literaria que permite al especialista concentrarse en unidades menores o mayores que el texto: recursos, temas, tropos —o géneros y sistemas, pero es necesario que este abordaje sea complementado por una lectura más cercana que focalice textos individuales y penetre en los pormenores de esos textos.

\section{Referencias bibliográficas}

Bernheimer, C. (org.) (1995). Comparative Literature in the Age of Multiculturalism. Baltimore: John Hopkins Univ. Press.

Buescu, H. (2014). «Inventar a ler. Literatura-mundo em português» en Abdala Junior, B. (org.). Estudos comparados: Teoria, Crítica e Metodologia. Cotia, SP: Ateliê Editorial, pp. 43-84.

Coutinho, E. (2013). Literatura Comparada: reflexóes. São Paulo: Annablume, 2013.

Coutinho, E. \& Carvalhal, T. (orgs.) (2011). Literatura Comparada: textos fundadores. $2^{\mathrm{a}} \mathrm{ed}$. Rio de Janeiro: Rocco.

Damrosch, D. (2003). What is World Literature? Princeton: Princeton Univ. Press. 
Nuevos rumbos del comparatismo: literatura comparada y literatura-mundo $\cdot$ E. Coutinho

Heise, U. (org.) (2017). Futures of Comparative Literature. ACLA State of the Discipline Report. New York: Routledge.

Jовıм, J.L. (org.) (2017). A circulaçāo literária e cultural. Oxford: Peter Lang Ltd.

LongXI, Z. (2017). "Aspects of World Literature» en Letteratura e Letterature. Pisa/Roma: Fabrizio Serra Editore, pp. 59-70.

Moretti, F. (2013). Distant Reading. Londres: Verso.

Saussy, H. (org.) (2006). Comparative Literature in the Age of Globalization. Baltimore: John Hopkins Univ. Press.

SpIvak, G. (2003). Death of a Discipline. N. York: Columbia Univ. Press. 